\title{
Les textes courts de Claude Simon : tentative de catégorisation
}

\section{Alastair B. Duncan}

\section{(2) OpenEdition}

1 Journals

Édition électronique

URL : http://journals.openedition.org/ccs/817

DOI : $10.4000 /$ ccs. 817

ISSN : 2558-782X

Éditeur :

Presses universitaires de Rennes, Association des lecteurs de Claude Simon

\section{Édition imprimée}

Date de publication : 31 décembre 2011

Pagination : 45-59

ISBN : 9782354121464

ISSN : $1774-9425$

\section{Référence électronique}

Alastair B. Duncan, «Les textes courts de Claude Simon : tentative de catégorisation », Cahiers Claude Simon [En ligne], 7 | 2011, mis en ligne le 21 septembre 2017, consulté le 01 mai 2019. URL : http:// journals.openedition.org/ccs/817; DOI : 10.4000/ccs.817 


\title{
Les textes courts de Claude Simon : tentative de catégorisation
}

\author{
Alastair B. DUNCAN \\ Université de Stirling
}

Les textes courts de Claude Simon comprennent des genres très variés : conférences, articles de presse, fragments de fictions. Pour les besoins de cet article, nous nous limiterons uniquement au dernier de ces genres; et parmi les textes qui appellent un jugement esthétique, nous exclurons ceux publiés en librairie - Femmes $/ L a$ Chevelure de Bérénice (1966/1983) et Orion aveugle (1970) - et les deux textes repris intégralement dans le volume posthume Archipel et Nord (2009). Dans le tableau annexe à cet article, nous avons mis en rapport avec des romans précis les textes courts qui correspondent à notre définition restrictive. Plusieurs constatations s'imposent. À une exception près, tous les textes courts ont été repris sous une forme ou une autre, parfois partiellement, parfois sous forme très modifiée, à l'occasion dans deux romans différents. Egalement à une exception près, tous sont des avant-textes. À partir de La Route des Flandres (1960), seuls Triptyque (1973), L'Invitation (1987) et Le Tramway (2001) n'ont pas été précédés par des avant-textes. Nous tenterons ici de ranger ces textes dans plusieurs catégories pour voir quels enseignements peuvent en être tirés, même si les frontières 
entre les catégories sont floues. En cours de route, nous nous permettrons de regarder certains textes d'un peu plus près que d'autres.

\section{Les « pré-textes"}

Dans une première catégorie, on peut ranger des textes dont la publication précède de peu la parution du roman dans lequel ils sont largement repris. Ils sont parfois présentés comme des " extraits". Ce sont à la limite de "bonnes feuilles ", souvent sollicitées par des directeurs de revue ou par des éditeurs d'ouvrages collectifs et destinées à donner un avant-goût du roman à venir. On pourrait citer, avant la publication de La Route des Flandres, "La poursuite "; avant Le Palace (1962), "Funérailles d'un révolutionnaire assassiné ", "Inventaire " et "L'Attentat "; avant L'Acacia (1989), "Parenthèse " et "Fragment "; avant Le Jardin des Plantes (1997), "Les jardins publics ", "Lecture publique d'une pièce de théâtre. Fragment d'un texte ", et « Polygone ». Beaucoup de ces textes ne subissent que de légères modifications avant de paraître dans le roman qui les accueille. Des coquilles sont corrigées, des lourdeurs et d'inutiles répétitions éliminées. Parfois, les modifications peuvent être plus importantes. Le texte «Propriété des rectangles » est publié en 1971 peu de temps avant la parution des Corps conducteurs (1971). Les fragments de ce collage sont distribués autrement dans la version romanesque. Les sept premières pages sont reprises dans l'ordre et presque mot à mot entre les pages 130 et $146 \mathrm{du}$ roman. Toutes les phrases utilisées dans les cinq pages qui suivent figurent également dans le roman mais souvent dans un ordre différent et éparpillées entre les pages 148 et 196. Simon a ajouté dans le roman - ou supprimé pour le texte à paraître en revue - des descriptions d'images pornographiques vues dans une vitrine de magasin.

L'écrivain a lui-même commenté un autre texte bref du même type, "Les Géorgiques », publié dans La Nouvelle Revue française en 1978.

Le livre en fait se déroule sur quatre niveaux. Dans un fragment de vingt-cinq pages, il n'était pas possible de les conserver. Je les ai donc réduits [...] Il m'a semblé que dans ce texte (je dis qu'il m’a semblé... A vous de me dire si j'ai eu tort...) le contraste entre les deux typogra- 
phies (l'emploi alterné des caractères courants et des petites capitales) faisait ressortir le contraste entre la figure colossale, presque mythique, de l'ancêtre et sa descendance déclinante. ${ }^{1}$

"Les Géorgiques » correspond assez précisément à une partie du chapitre III du roman. Il évoque la vie de cette famille déclinante : la vieille femme et le garçon, tous deux hantés par le souvenir de leur ancêtre illustre. La grande différence, c'est qu'en remaniant ce passage pour le roman, Simon y colle de longs extraits de lettres de L.S.M. ; dans le texte court, figurent à la place de ces lettres, en majuscules, et bien plus fréquemment, des informations biographiques sur Lacombe Saint-Michel, extraites en partie, comme l'a signalé Ralph Sarkonak, d'un livre de A. Dry, Soldats ambassadeurs sous le Directoire, an IV-an VIII. ${ }^{2}$ En commentant le texte court, Simon jette une lumière intéressante sur Les Géorgiques (1981) : "Le livre, dit-il, se déroule sur quatre niveaux ». On se serait peut-être attendu au mot "série " - mot que l'écrivain employait pour parler des Corps conducteurs et de Triptyque. Les Géorgiques, on le sait, se compose de trois " séries " principales : celle de L.S.M., celle du cavalier de l'an 40, et celle de l'engagé volontaire en Espagne. Le mot " niveau " propose une autre façon de penser l'organisation du roman, en fonction des divers foyers de perception mis en place. Les quatre " niveaux » que Claude Simon évoque seraient peut-être : la perception englobante du narrateur/écrivain ; les lettres et les documents du $18 \mathrm{e}$ siècle ; la perspective d'O. sur sa propre histoire ; les multiples foyers de perception secondaires (le cavalier/visiteur/garçon et, plus passagèrement, Batti et Jean-Marie L.S.M.). Dans le texte court, Simon a " réduit " les foyers de perception en omettant entièrement le cas de O. et en substituant Dry aux textes du $18^{\mathrm{e}}$ siècle. Le mot " réduit " peut d'ailleurs surprendre. Mais c'est effectivement comme si le texte court, malgré sa date de publication en 1978, donc trois ans avant

1. «Entretien avec Jo van Apeldoorn et Charles Grivel, 17 avril 1979, de Claude Simon ", dans Ecriture de la religion, écriture du roman, Charles Grivel (dir.), Lille, Presses Universitaires de Lille, 1979, p. 101-102.

2. Paris, Plon, 1906. Dry était le pseudonyme d'Alfred Fleury. Voir Ralph Sarkonak, "Comment fait-on un cocktail simonien ? Ou, Les Géorgiques relues et corrigées ", Romanic Review, 81, n² 2, 1990, p. 239. 
celle du roman en 1981, avait été écrit ou modifié après la rédaction du roman. Il ne faut pas prendre cette idée à la lettre : Simon a beaucoup retravaillé le roman entre 1978 et 1981. Mais l'emploi du verbe " réduire " indique qu'au moment de publier "Les Géorgiques " Simon travaillait déjà avec ces quatre " niveaux $»^{3}$.

\section{Des « après-textes" ?}

Étant donné le souci de la forme et le perfectionnisme de Claude Simon, il est tout à fait possible que certains avant-textes paraissant peu avant la publication d'un roman aient été modifiés pour paraître en revue. Dans ces cas, on aurait affaire non pas à des " avant" mais à des après-textes. Un seul texte appartient sans aucun doute à cette catégorie : "Lieu ", version des premières pages de Triptyque, revues après coup pour paraître dans L'Humanité en 1977. Comme dans le cas des "Géorgiques ", Simon procède par simplification. Dans le journal, le titre "Lieu » recouvre, en plus de la fiction, une introduction où Simon parle de son attachement aux paysages du Jura et de l'influence exercée sur cette petite réécriture de Triptyque par un tableau de Jean Dubuffet ${ }^{4}$. La simplification du texte va dans le sens indiqué par cette note. Dans Triptyque des présages de destruction et de mort courent en filigrane le long des premières pages. Dans "Lieu " Simon exclut pour une large part ces éléments sombres; le texte présente une image idyllique du Jura, pays de ses tantes paternelles et des vacances heureuses de son enfance ${ }^{5}$.

\section{Des textes explorateurs}

Certains textes courts de Simon sont écrits comme des essais, des tentatives d'approche, des moyens de trouver le bon rythme, le

3. Simon a beaucoup travaillé la partie " O. » dans les premiers mois de 1978.

4. Il s'agit des Riches fruits de l'erreur (1963). Les deux textes s'accompagnent de reproductions de deux autres tableaux de Dubuffet : Vache au nez subtil et Jardin de Blèche grignotte.

5. Pour plus de détails, voir A. B. Duncan, "Claude Simon, Michel Deguy et Triptyque", dans Transports. Les Métaphores de Claude Simon, Irène Albers et Wolfram Nitsch (dir.), Frankfurt, Peter Lang, 2006, p. 40-41. 
meilleur angle d'attaque pour un roman à venir. Dans cette catégorie, on pourrait mettre "Correspondance ", paru trois ans avant Histoire (1967). Simon y décrit des cartes postales envoyées par son père à la jeune femme qui allait devenir sa mère. A partir de ces descriptions, l'écrivain évoque la jeunesse de sa mère et l'histoire d'amour à distance entre ses parents. "Correspondance » est une tentative réussie. Ce texte représente un moment crucial dans la genèse d' Histoire, moment où " la sauce prend ". Simon tient désormais la charpente du roman. Travaillés et retravaillés, des éléments de "Correspondance " figurent au premier et au dernier chapitre d' Histoire.

Des textes qui précèdent Les Géorgiques fournissent d'autres exemples de cette catégorie : par exemple "Le Régicide " ou même "Le Général ", encore que ce dernier texte soit repris presque intégralement. Rien d'étonnant à la prolifération de textes avant Les Géorgiques. Simon a sérieusement travaillé le projet de ce roman pendant plus de huit ans, entre 1972 et 1981 . Et le titre de son "Essai de mise en ordre de notes prises au cours d'un voyage en Zeeland (1962) et complétées » fait croire que l'idée d'écrire quelque chose à partir de la vie du son ancêtre, le général Jean-Pierre Lacombe Saint-Michel, le tentait depuis au moins 1962. Dans le roman, Simon abandonne le fil conducteur de cet " Essai de mise en ordre " : un voyage à travers les Pays-Bas ${ }^{6}$; il retient et développe considérablement l'emploi de citations et le thème de la femme aimée et perdue. Deux autres avant-textes des Géorgiques connaissent des sorts contrastés. "Progression dans un paysage enneigé ", publié en 1976, est présenté par Jean-Pierre Vidal, comme "un inédit [...] extrait d'un roman en cours d'écriture ${ }^{7}$. Pendant des années, Simon cherche à caser les trois fragments de ce texte dans son roman, ensemble, ou séparément en tant que prologues à différentes parties du livre. Il n'y parvient pas. Des images et des tournures de phrases semblables trouvent une place dans Les Géorgiques, mais aucune des trois descriptions d'un homme à cheval dans un paysage enneigé.

6. Voir Sjef Houpermanns, «Par terre et par mer : voyages parallèles (du 'Voyage en Zeeland' aux Géorgiques) ", La Revue des lettres modernes, Claude Simon, 5, JeanYves Laurichesse (dir.), 2008, p. 81-96.

7. Études littéraires, 9, n 1, 1976, p. 217. Repris dans Les Cabiers Claude Simon, $\mathrm{n}^{\circ} 3,2007$, p. 13. 
En revanche, un sort extraordinaire attend le texte " Deux personnages ». Publié en 1973, premier des avant-textes des Géorgiques à être exposé au public, c'est ce fragment qu'en fin de compte Simon choisit intégralement comme prologue du roman. La première phrase écrite subsiste souvent comme incipit des romans de Simon : "c'est le cas, dit-il, de La Route des Flandres, d'Histoire, de L'Herbe [1958], du Palace, de La Bataille de Pharsale [1969] [...], des Géorgiques " . Mais le cas des Géorgiques est exceptionnel vu l'évolution considérable de l'art de l'écrivain dans les années soixante-dix. Par sa forme, "Deux personnages " porte la trace de ses origines au début de la décennie. Simon décrit et analyse des tableaux dans Les Corps conducteurs de 1971, surtout Paysage avec Orion aveugle ${ }^{9}$. Le prologue des Géorgiques est de la même facture : description et commentaire précis et détaillé d'une œuvre d'art, syntaxe et ponctuation classiques, ton objectif. Par la suite, regrettant que Les Corps conducteurs ait donné prise à des interprétations centrées sur le personnage de l'homme malade, Simon projette d'écrire dans Triptyque un roman «irréductible à tout schéma réaliste, c'est-à-dire un roman où les rapports entre les différentes 'séries' (ou 'ensembles') ne relèveraient pas d'un quelconque enchaînement ou déterminisme d'ordre psychologique " ${ }^{10}$. Les "séries" de Triptyque impliquent des personnages et des histoires des plus banals, sans plus d'intérêt que des faits divers. Tout est dans la manière de les écrire et dans le chevauchement et l'interpénétration des descriptions. Mais déjà dans "Deux personnages " un autre aspect de l'art de Simon revoit le jour. Les personnages, quoiqu'anonymes, sortent de l'ordinaire. Des indications d'un uniforme - « tunique bleu roi, au col montant et rouge [...] ornée d'épaulettes aux franges dorées ${ }^{11}$ rattachent l'homme plus âgé à l'Histoire. Le narrateur se livre à des spéculations sur les gestes et les motivations des personnages; il se pose des questions sur leurs rapports. Simon revient ainsi d'une fa-

8. Claude Simon dans Lucien Dällenbach, Claude Simon, Seuil, 1988, p. 171172.

9. Les Corps conducteurs, Minuit, 1971, p. 50-51, 58-59, 65-66, 85-86 et surtout p. 77-79.

10. "Claude Simon, à la question", dans Claude Simon : analyse, théorie, Jean Ricardou (dir.), U.G.E., coll. «10/18», 1975, p. 424.

11. «Deux personnages », Art Press, février 1973, p. 15. 
çon renouvelée à des préoccupations de la fin des années cinquante. Comme Le Vent, "Les Géorgiques " est une " tentative de restitution ». Dans ce texte, comme dans La Route des Flandres, s'expriment à la fois la volonté et l'impossibilité de savoir.

Un autre texte, publié quelque quinze ans plus tôt, en 1958, peut également compter comme tentative d'approche. "Le Cheval » est une première version de l'histoire de paysans qui reparaît dans $\mathrm{La}$ Route des Flandres. La nouvelle prend la forme d'un conte de fées qui tourne en cauchemar. A la guerre, face à la mort toute proche, le narrateur cherche quel sens il peut donner à la vie. Avec ses camarades de régiment, chevauchant à travers la nuit et la pluie, désorienté par la fatigue, il quitte le monde ordinaire. À l'arrivée dans un hameau perdu, il aperçoit une jeune femme : " une sorte d'apparition non pas éclairée par la lanterne mais bien plutôt lumineuse par ellemême [...] non une femme, mais l'idée même, le symbole de toute femme et de toute paix, tel que, dans notre célibat forcé, notre jeunesse frustrée, affamée, nous pouvions le concevoir ${ }^{12}$. Il part donc à la recherche de cette femme entrevue, mais en vain : la piste se perd dans un imbroglio sexuel ; la seule femme qu'il arrive à rejoindre n'est pas « elle, cette blancheur, cette espèce de suave et tiède apparition [...] mais pour ainsi dire son contraire, ou plutôt sa négation, ou plutôt sa corruption, la corruption même de l'idée de femme, de grâce, de volupté, son châtiment ». D'où le retournement du conte de fées en mauvais rêve : «moi le chevalier, le conquérant botté venu délivrer au fond de la nuit, séduire, enlever la liliale princesse, et au moment où je croyais parvenir jusqu'à elle, la prendre dans mes bras, les refermant, enserrant une horrible et goyesque vieille $»^{13}$.

Dans La Route des Flandres, Simon garde l'aspect cauchemardesque de l'histoire paysanne, tout en faisant éclater les éléments et en brouillant irrémédiablement les fils de l'intrigue. Cette histoire entre en résonance avec les autres histoires du roman. La recherche auprès de la femme d'une consolation, d'un contrepoids à la mort se projette sur Corinne. La désillusion de Georges n'en est que plus grande. Et le roman hérite également d'un autre motif de la nouvelle, d'un autre

12. "Le Cheval ", Les Lettres nouvelles, $\mathrm{n}^{\circ}$ 57, 1958, p. 175.

13. «Le Cheval ", Les Lettres nouvelles, $n^{\circ} 58,1958$, p. 385. 
espoir de conjurer la mort. Tout comme la jeune femme proposait " un symbole de toute paix ", l'œil d'un cheval mourant semble entrevoir et refléter « une vision intérieure, une réalité plus réelle que le réel, plus paisible que l'harassante agitation des humains, plus stable que... " ${ }^{14}$. Comme dans La Route des Flandres, ce bref aperçu d'une transcendance sera vite balayé, entre autres raisons, par l'intervention brutale du copain du narrateur : "A part la certitude de crever, dit Maurice, est-ce que tu as une seule idée de la vie à peu près stable ? " ${ }^{15}$. Mais «Le Cheval » se distingue de La Route des Flandres en mettant la transcendance aperçue dans l'œil du cheval en rapport explicite avec la perte ou plutôt la disparition de la foi chrétienne :

"Ouais, pensai-je (réagissant, me secouant, quittant le pied de l'échelle pour aller fouiller dans la sacoche de ma selle où j'avais gamelle et car) ouais : comme une poignée de billes, de dés. Voilà. Rien de plus. Une poignée de dés dans la main... ". Mais dans la main de qui au fait ? Car je ne croyais pas en Dieu et il y avait même longtemps que j'avais cessé d'y croire, si tant est que j'y eusse jamais cru, si tant est que je ne me fusse pas contenté un jour (vers l'âge de treize ou quatorze ans) de prendre conscience du fait qu'en réalité je n'y avais jamais cru, que j'avais répété les paroles que m'avaient d'abord apprises ma mère et après elle les prêtres sans que cela correspondît à rien d'autre que des mots et la peur d'une punition (et bien plus humaine que divine, et bien plus temporelle que de l'esprit), de sorte que contrairement à ce qui se passe paraît-il habituellement dans les cas semblables il n'y eut jamais pour moi ni crise ni drame, Mais ceci est une autre histoire et la seule question alors était que ne croyant à rien, ni à Dieu ni au... Au fait, peut-être au Diable. Mais mieux valait...

"Alors, pensai-je, disons : dans la main du Seigneur. Comme une poignée de dés dans la main du Seigneur, et qu'importe son nom, qu'il soit celui de la Guerre, du Mal, du Bien, le Seigneur donc agitant son poing fermé à hauteur de son oreille, un peu en arrière de l'épaule, comme font les joueurs de poker dice assis sur les hauts tabourets des bars, écoutant le bruit familier, le bruit hasardeux, excitant, de l'ivoire, de nos os entrechoqués, puis lançant les petits cubes, les regardant rouler, se culbuter, glisser, s'immobiliser enfin sur le tapis vert, les verts pâturages, les vertes prairies de la guerre, et se penchant, curieux, pour regarder. Ouais. En attendant qu'est-ce qu'ils foutent ces types de la soupe ?" ${ }^{16}$

14. Ibid., p. 380.

15. Ibid., p. 380.

16. Ibid., p. 383-84. 
D'après ce passage, le narrateur sort de la foi sans crise, sans drame. Nous savons que l'écrivain, élevé dans la foi catholique, est passé à un matérialisme foncier. Comme l'a remarqué Jean-Yves Laurichesse à propos de "Cendre ", " le bon usage de la maladie " consiste pour Simon "non comme chez Pascal [à] s'élever vers la transcendance, mais tout au contraire [à] approfondir l'immanence $»^{17}$. Pour lui, la vie des sens est suffisante; face à la mort, il développe un plus grand attachement à l'expérience du voir, de l'entendre et du sentir, à ce qu'il appelle dans Le Jardin des Plantes « cette déchirante et mélancolique avidité avec laquelle le condamné regarde autour de lui le monde $»^{18}$.

Et pourtant on pourrait aussi avancer que, sorti de la foi, Simon en reste imprégné. Au deuxième paragraphe de la citation, le Seigneur est imaginé en joueur de poker. On sait l'importance du thème du hasard dans l'œuvre : affirmer le hasard en tant que principe, c'est nier la Providence. Mais la question d'un sens ultime, transcendant reste posée. La foi reste en tant que creux - creux à remplir, absence que Simon sera toujours tenté de compenser. Dans "Le Cheval» cette tentation prend la forme du contraire du Dieu : " ne croyant à rien, ni à Dieu ni au... Au fait, peut-être au Diable ». Bien sûr, il n’y croit pas, mais l'œuvre sera riche en images qui laissent flotter l'éventualité d'une force maligne à l'œuvre dans le monde. Dans le dernier paragraphe de la citation, Simon fait appel à plusieurs termes abstraits pour la nommer : la Guerre, le Mal, le Bien. Dans certains romans, cette possibilité d'une puissance transcendantale malveillante aura un autre nom : l'Histoire. C'est sous cette appellation qu'elle apparaît par exemple dans Les Géorgiques : personnification parodique du mal : "Ou peut-être, après avoir frappé un premier coup, épouvantée elle-même, l'Histoire s'accordait-elle un répit " ${ }^{19}$. Ailleurs, par exemple dans Le Tramway, des comparaisons font que certains moments semblent s'ouvrir sur un au-delà, " une réalité plus réelle

17. "'Cendre' : un avant-texte lointain du Jardin des Plantes ", Cahiers Claude Simon, $\mathrm{n}^{\circ} 2,2006$, p. 140.

18. Claude Simon, Euvres, Gallimard, coll. «Bibliothèque de la Pléiade », 2006, p. 1124.

19. Les Géorgiques, Minuit, 1981, p. 131. 
que le réel $»^{20}$, même si l'élan de transcendance est presque toujours freiné par un " comme si ». Les ombres des fanaux, éclairant le visage des pêcheurs, " conféraient cet on ne savait quoi de mystérieux et de sacré qui, dans les tableaux et les illustrations du catéchisme représentant la Nativité, transforme et magnifie ceux du berger $»^{21}$.

\section{Des avant-textes lointains}

Certains textes, qui n'ont été écrits en vue d'aucun roman, restent parfois pendant des années, voire des décennies, des pièces détachées dans l'atelier de Claude Simon, attendant de trouver un usage. "Matériaux de construction ", écrit à la fin des années cinquante et publié en 1960, est à cet égard un texte emblématique et par son titre et par ce qu'il devient. La première de ses trois parties évoque sans la nommer la ville d'Arbois : elle ne sera jamais reprise. La deuxième partie décrit le bureau encombré d'un personnage anonyme et l'" odeur douceâtre et entêtante d'alcool à brûler de sucre et de moût chauffé ${ }^{22}$ qui y règne ; quelques années plus tard, Simon retravaille ce passage et fait de ce personnage l'oncle Charles d'Histoire. La troisième partie décrit deux hommes de "type méditerranéen " ${ }^{23}$ aperçus sur un quai de gare à Narbonne. De cette première tentative d'écrire un incident survenu en 1958 va sortir Le Palace ${ }^{24}$.

D'autres avant-textes d'Histoire appartiennent à cette même catégorie : "Le Candidat " (1958), "Mot à mot " (1959) et "Comme du sang délayé » (1960). "Sous le kimono » (1961) est exploité dans La Bataille de Pharsale de 1969. Le laps de temps entre textes fragmentaires et romans devient de plus en plus grand. Dans L'Acacia, en 1989, Simon reprend des éléments de "Babel », publié en 1955, trente-quatre ans plus tôt. Entre "Cendre " (1959) et Le Jardin des Plantes trente-huit ans se sont écoulés. Cela confirme ce que nous sa-

20. "Le Cheval », Les Lettres nouvelles, n 58, 1958, p. 380. La Route des Flandres, Gallimard, coll. "Bibliothèque de la Pléiade ", 2006, p. 283.

21. Le Tramway, Minuit, 2001, p. 51.

22. "Matériaux de construction ", Les Lettres nouvelles, n 9, déc. 1960, p. 116.

23. Ibid., p. 121.

24. Voir Jean-Yves Laurichesse, "Notes sur 'Matériaux de construction' ", Cahiers Claude Simon, $\mathrm{n}^{\circ}$ 4, 2008, p. 19-27. 
vons déjà de la façon de travailler de Claude Simon. L'image de l'atelier est plus qu'une métaphore. Simon avait autour de lui dans son bureau, dans son appartement, des dossiers de textes publiés et non publiés. Il avait aussi une mémoire fabuleuse. Dans "Cendre ", il évoque la maladie à laquelle il a failli succomber au début des années cinquante. Il y revient dans Le Jardin des Plantes; les circonstances sont les mêmes, les images se ressemblent, la cadence des phrases aussi, mais tout s'exprime autrement. Simon a-t-il relu "Cendre " avant d'écrire les pages du Jardin des Plantes? C'est possible. Mais l'écrivain avait aussi cela en tête. Chez Simon, l'écriture du souvenir se compose toujours, entre autres, du souvenir du déjà écrit.

Un texte du même genre éclaire en partie, comme "Le Cheval ", des œuvres à venir. "Babel ", publié en 1955, est, comme " Le Cheval ", une nouvelle qui respectent des normes de structure presque classique : unité de lieu, de temps et d'action. Le thème de "Babel » est politique. Il s'agit d'une fable sur l'impossibilité de faire la révolution. Toutes les nations de la terre viennent à Moscou pour admirer la construction de la nouvelle société. Mais en vain : une histoire de jalousie sexuelle démontre que l'homme nouveau soviétique ne dépasse pas l'homme ancien. A l'origine de la nouvelle est le séjour de Simon à Moscou en 1937. Quand il reprend le souvenir de ce séjour et de la nouvelle dans L'Acacia dans les années quatre-vingts, il laisse tomber la fable et sa morale. Il garde un autre aspect du texte, mais de façon très différente. Le contraste ressort de cet extrait de Babel :

Aujourd'hui, en regardant en arrière, tout cela semble si lointain, un peu ridicule même, comme ces cartes de géographie dans les Atlas des anciens programmes, avec leurs pays aux teintes fanées, leurs frontières absurdes. Mais alors nous ne savions pas encore, nous qui avions entre vingt et trente ans dans cette Europe mal raccommodée de l'entre-deux-guerres, essayant maladroitement de devenir des hommes, ou du moins quelque chose qui dans notre idée correspondît à ce mot, pris entre ces mythes nouveaux, enivrants et, pensions-nous, irréductibles, qui avaient nom Verdun ou Révolution d'Octobre, proposés à nos juvéniles enthousiasmes sans qu'aucun de ceux qui prétendaient nous servir de guides, les aventuriers déclamatoires, les graves mentors aux yeux rusés, à la sagesse mercantile et vaniteuse, ne prît la peine de nous dire (mais les aurions-nos crus, les eussions-nous écoutés, nous qui dévorions avidement leurs livres où les sempiternelles pacotilles pêlemêle, le fracas des armes, l'ivresse du néant, les creux raffinements de 
lettrés, se présentaient avec la séduisante estampille d'un visage de Lama ou d'un Orient agonisant, ratiocineur et opiacé), sans qu'aucun d'eux donc, ne prît la peine de nous dire que ce après quoi nous courions était déjà loin derrière nous et que nous ne devions jamais l'attraper, si tant est naturellement que cela existât, ni plus ni moins que de jeunes chiens s'essoufflant à la poursuite de leur queue. ${ }^{25}$

Dans la nouvelle, Simon situe toute une génération de jeunes dans un contexte historique précis, celui de l'entre-deux-guerres. Son ironie vise "pêle-mêle " les idéologies concurrentielles et les divers maîtres à penser des années trente. Un seul mot, "Verdun ", indique éventuellement le nationalisme des ligues d'anciens combattants telles les Croix-de-feu, tout comme " la Révolution d'Octobre » évoque le marxisme. Dans les " creux raffinements de lettrés " et " le visage de Lama » on est tenté de reconnaître un portrait peu flatteur de Gide. A travers " les aventuriers déclamatoires ", "le fracas des armes, l'ivresse du néant ", et le visage "d'un Orient agonisant " transparaissent sans aucun doute la personne et l'œuvre de Malraux allant de La Tentation de l'occident à la figure de Tchen dans La Condition humaine ${ }^{26}$.

Trente ans plus tard, Simon prend encore plaisir à écrire des tirades dirigées contre des cibles qu'il ne désigne que par périphrases - comme il le fait en parlant des dirigeants soviétiques dans L'Invitation, cet autre retour de l'URSS. Mais, dans L'Acacia, l'ironie a une autre cible. Elle vise la naïveté allant jusqu'à la mauvaise foi d'un jeune qui essaye " maladroitement " de devenir un homme. Incorrigible joueur de rôles - anarchiste, peintre cubiste, rentier -, il se sert des banques tout en portant « la carte d'un parti politique dont le but déclaré était de supprimer les banques en même temps que leurs clients ${ }^{27}$. Dans L'Acacia le jeune homme ne fait pas explicitement partie d'une génération. Il n'est pas présenté comme typique. Simon passe plus directement du particulier à l'universel.

Toute tentative de catégorisation finit en échec : elle laisse des cas douteux et des exceptions. Deux avant-textes d'Histoire sont à

25. "Babel ", Les Lettres nouvelles, $\mathrm{n}^{\circ}$ 31, 1955, p. 391-92.

26. Grasset, 1926 ; Gallimard, 1936.

27. L'Acacia, Minuit, 1989, p. 192. 
cheval entre les catégories. «Des roches striées vert pâle parsemées de points noirs " est publié trois ans avant la parution d'Histoire quand Simon cherche encore sa voie. Mais ce fragment lui semble réussi : il le reprend presque intégralement. "La Statue " paraît également en 1964. Ce texte aussi est repris dans Histoire, sous forme éclatée. L’exception est le texte que Simon rédige à la demande de Maeght pour inspirer une lithographie de Pierre Alechinsky. Ce texte qui devient le prologue de Leçon de choses ne fut pas écrit en vue de ce roman. Ayant décrit la pièce en réfection, ayant esquissé la possibilité d'écrire un roman à partir de cette description, Simon relève le défi qu'il s'est lancé : " une fois envoyé ce texte à Maeght, je me suis dit : 'Mais, puisque tu as écrit ça, fais-le !... Concrétise-le !...' " ${ }^{28}$. Ainsi le ponctuel et le hasard contribuent aux méandres des sentiers de la création.

\section{Les textes courts et les romans de Claude Simon}

"Le Cheval », Les Lettres nouvelles, n 57, 1958, p. 169-89; et ${ }^{\circ}$ 58, 1958, p. 379-394.

"La Poursuite ", Tel Quel, n 1, printemps 1960, p. 49-60.

\section{La Route des Flandres, 1960.}

"Matériaux de construction [...]», Les Lettres nouvelles, nouv. série, $\mathrm{n}^{\circ}$ 9, déc. 1960, p. 112-122.

"Funérailles d'un révolutionnaire assassiné », Médiations, $\mathrm{n}^{\circ} 4$, 1961, p. 11-24.

"Inventaire », Les Lettres nouvelles, n 22, février 1962, p. 50-58. "L'Attentat ", La Nouvelle Revue française, 19, n 111, 1962, p. 431-452.

\section{Le Palace, 1962.}

"Mot à mot ", Les Lettres nouvelles, nouv. série, n6, 1959, p. 6-10 ; et Cahiers Claude Simon, n 5, 2009, p. 11-15.

"Le Candidat ", Arts, n 698, 26 novembre-2 décembre 1958, p. 3 ; et Cahiers Claude Simon, n 6, 2010, p.11-15.

28. Jean-Paul Goux et Alain Poirson, «Un homme traversé par le travail. Entretien avec Claude Simon ", La Nouvelle Critique, n 105, juin-juillet 1977, p. 35-36. 
"Matériaux de construction ", Les Lettres nouvelles, $\mathrm{n}^{\circ}$ 9, déc. 1960, p. 112-122 ; et Cahiers Claude Simon, n 4, 2008, p. 19-27. "Comme du sang délayé ", Les Lettres françaises, 1-7 déc., 1960, p. 1 et 5 ; et Cahiers Claude Simon, n 6, 2010, p.19-22.

"Des roches striées vert pâle parsemées de points noirs ", Les Lettres nouvelles, juin-août 1964, p. 53-68.

"La Statue », Mercure de France, vol. 352, n 1213, 1964, p. 393409.

"Correspondance ", Tel Quel, n 16, hiver 1964, p. 18-32.

Histoire, 1967.

"Sous le kimono », Les Lettres françaises, 19-25 janvier 1961, p. 5.

\section{La Bataille de Pharsale, 1969.}

"Propriété des rectangles ", Tel Quel, n 44, 1971, p. 3-16 Les Corps conducteurs, 1971.

\section{Triptyque, 1973.}

"Lieu ", L'Humanité, 9 décembre 1977, p. 2 ; et Les Triptyques de Claude Simon ou l'art du montage, Mireille Calle-Gruber (dir.), Presses Sorbonne nouvelle, 2008, p. 95-99.

"Les langues pendantes de papier... ", texte de Claude Simon, lithographie de Pierre Alechinsky, édition limitée à 40 exemplaires, dans la série "Placards ", Maeght, 1974.

Leçon de choses, 1975.

«Deux personnages », Art Press, février 1973, p. 14-15.

"Essai de mise en ordre de notes prises au cours d'un voyage en Zeeland (1962) et complétées ", Minuit, n 3, mars 1973, p. 1-18 ; et Cahiers Claude Simon, n 1, 2005, p. 17-34.

"Progression dans un paysage enneigé ", Etudes littéraires, 9, $\mathrm{n}^{\circ}$ 1, 1976, p. 217-221 ; et Cahiers Claude Simon, n 3, 2007, p. 13-17 (non repris).

"Le Général », Art Press, été 1977, p. 28-29.

"Le Régicide », La Nouvelle Critique, juin-juillet 1977, p. 45-46. 
"Les Géorgiques ", La Nouvelle Revue française, t. 52, n 308, 1978, p. 1-27.

\section{Les Géorgiques, 1981.}

«Babel », Les Lettres nouvelles, $\mathrm{n}^{\circ}$ 31, 1955, p. 391-413.

"Parenthèse ", Revue de la Bibliothèque Nationale, printemps 1985, p. 3-6.

"Fragment ", dans Claude Simon : New Directions, Alastair B. Duncan (dir.), Edinburgh, Scottish Academic Press, 1985, p. 2-11.

\section{L'Acacia, 1989.}

"Cendre ", La Revue de Paris, mars 1959, p. 79-82 ; et Cahiers Claude Simon, no 2, 2006, p. 133-137.

"Les Jardins publics», dans Les Sites de l'écriture: Colloque Claude Simon, Mireille Calle-Gruber (dir.), Paris, Nizet, 1995, p. 25-37. "Lecture publique d'une pièce de théâtre. Fragment d'un texte ", L'Infini, n 56 , hiver 1996, p. 3-10.

"Polygone ", Cahiers de la Bibliothèque littéraire Jacques Doucet, $\mathrm{n}^{\circ} 1,1997$, p. 11-15.

Le Jardin des Plantes, 1997. 